EdUCACIÓN VETERINARIA

\title{
ESTRATEGIAS PEDAGÓGICAS PARA LA MEJORA DE LAS HABILIDADES COGNITIVAS Y EMOCIONALES DE LOS ALUMNOS DE LA FACULTAD DE MEDICINA VETERINARIAY ZOOTECNIA, UPCH, PERÚ
}

\author{
Teaching Strategies for Improving Cognitive and Emotional Skills of the \\ Students at the Veterinary Medicine and Animal Husbandry Faculty, \\ UPCH, PERU
}

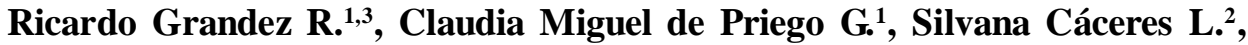 \\ Ricardo Rojas M. ${ }^{1}$, Elizabeth Hinostroza M. ${ }^{1}$
}

\section{Resumen}

El documento presenta las acciones realizadas para modificar las características teóricas del curso de Medicina de Animales de Compañía, curso semestral obligatorio de la carrera de Medicina Veterinaria y Zootecnia de la Universidad Peruana Cayetano Heredia, Lima, Perú. Se organizó un curso práctico-teórico en búsqueda de mejorar los conocimientos básicos, habilidades y destrezas médico veterinarias; y asimismo, la mejora de las habilidades cognitivas y emocionales de los estudiantes mediante la innovación de metodologías y tecnologías educativas. Estas comprendieron: resolución de casos clínicos, desarrollo del pensamiento crítico y de la creatividad, revisión, interpretación y análisis de información científica, actividades contextualizadas en la práctica real, actividades de apoyo social y tecnologías de transferencia de la información científica, todo ello dentro de un marco referencial de la formación universitaria por competencias. La evaluación de las habilidades cognitivas y emocionales se realizó con una adaptación del Inventario de Cociente Emocional de Reuver Bar-On. Se realizó una encuesta a inicios y otra a fines del curso, participando 24 de los 30 alumnos matriculados. Los resultados muestran que la proporción de alumnos con habilidades cognitivas y emocionales calificadas como desarrolladas, adecuadas y por mejorar fue de 25,54 y $21 \%$, respectivamente, en la primera encuesta, mejorando a 54,33 y $13 \%$, respectivamente, en la segunda encuesta.

Palabras clave: habilidades cognitivas, habilidades emocionales, encuesta, tecnologías educativas, Perú

\footnotetext{
${ }^{1}$ Facultad de Medicina Veterinaria y Zootecnia, ${ }^{2}$ Oficina de Tutoría Académica y Consejería Psicológica, Universidad Peruana Cayetano Heredia, Lima, Perú

${ }^{3}$ E-mail: rgrandez@hotmail.com
}

Recibido: 16 de enero de 2013

Aceptado para publicación: 18 de marzo de 2014 


\section{Abstract}

The paper presents actions taken to modify the theoretical characteristics of the course of Medicine of Companion Animals, a mandatory course of the Veterinary Medicine and Animal Husbandry career at Universidad Peruana Cayetano Heredia, Lima, Peru. A practical-theoretical course was organized in pursuit of improving basic knowledge, skills and abilities in the veterinary field, and also for improving cognitive and emotional skills of students through innovative educational methodologies and technologies. These included resolution of clinical cases, critical thinking and creativity, revision, interpretation and analysis of scientific information, contextualized activities in actual practice, support of social activities and technology transfer of scientific information, all within a framework of university education by competences. The assessment of cognitive and emotional abilities was done by the Bar-On Emotional Quotient Inventory survey. A survey was conducted at the beginning and another at the end of the course involving 24 out of 30 registered students. The results show that the percentage of students with cognitive and emotional skills qualified as developed, adequate and to be improved was 25,51 and $21 \%$ respectively in the first survey, and improving to 54, 33 and $13 \%$ respectively in the second survey.

Key words: cognitive skills, emotional skills, survey, educational technologies, Peru

\section{ANTECEDENTES}

La sumilla del curso de Medicina de Animales de Compañía lo define como: «Curso de naturaleza teórico-práctico cuyo propósito es contribuir en la formación integral del Médico Veterinario mediante el estudio de las enfermedades de los diversos sistemas orgánicos de los animales de compañía, con énfasis en la etiología, las manifestaciones clínicas, la fisiopatología, los métodos diagnósticos y los procedimientos terapéuticos a realizarse en la solución médica de estos». Este es un curso semestral obligatorio de la carrera de Medicina Veterinaria y Zootecnia de la Universidad Peruana Cayetano Heredia (UPCH), Lima, Perú, el cual consta de tres créditos académicos (2 horas de teoría y 2 horas de práctica por semana).

El objetivo general del curso es integrar los conocimientos adquiridos y relacionarlos en la búsqueda de la solución médica de las diversas enfermedades que afectan a los animales de compañía, propiciando la capacidad analítica, la cual permita el constante aprendizaje de los conocimientos en la resolución de los problemas médicos necesarios para un adecuado desempeño profesional. Tiene como objetivos específicos: a) proponer y discutir la etiología, manifestaciones clínicas, fisiopatología, métodos diagnósticos y alternativas terapéuticas de las diversas enfermedades que afectan a los animales de compañía; b) investigar, analizar y discutir información científica relevante dirigida a la solución de los diversos problemas médicos de los animales de compañía; c) adquirir capacidades, habilidades y destrezas en la ejecución de procedimientos terapéuticos, diagnósticos clínicos rutinarios e imagenológicos mediante prácticas dirigidas; y d) ejercitar la realización de actividades de investigación mediante el estudio de prevalencias, casos clínicos, estudios retrospectivos y otras modalidades a partir de la casuística de la Clínica Veterinaria Cayetano Heredia $(\mathrm{CVCH})$ de la Facultad de Medicina Veterinaria y Zootecnia (FAVEZ). 
Este curso ha pasado por modificaciones paulatinas que lo han ido diferenciando de la enseñanza tradicional universitaria, guiándolo principalmente hacia un curso práctico-teórico clínico vivencial, con innovación de metodologías mixtas de enseñanza, tales como el aprendizaje basado en problemas (Dodd, 2007; Yoo y Park, 2014), resolución de casos clínicos (Andreu et al., 2004), desarrollo del pensamiento crítico y de la creatividad, revisión, interpretación y análisis de información científica (Kennedy, 2007), actividades contextualizadas en la práctica real, actividades de apoyo social (Folgueiras y Martínez, 2009), y tecnologías de transferencia de la información científica (Báez et al., 2006); todo esto dentro de un marco referencial de la formación universitaria por competencias (Larraín y González, s.f.).

El documento presenta las acciones realizadas para modificar las características teóricas de la asignatura a manera de poder organizar un curso práctico-teórico en búsqueda de mejorar los conocimientos básicos, habilidades y destrezas médico veterinarias; y asimismo, la mejora de las habilidades cognitivas y emocionales de los estudiantes participantes del curso mediante la innovación de metodologías y tecnologías educativas.

Bar-On (1997), Cortés et al. (2002) y Extremera et al. (2004) definen la inteligencia emocional como un conjunto de habilidades personales, emocionales y sociales y de destrezas que influyen en nuestra habilidad para adaptarnos y enfrentar las demandas y presiones del medio. Como tal, la inteligencia no cognitiva es un factor importante en la determinación de la habilidad para tener éxito en la vida, influyendo directamente en el bienestar general y en la salud emocional.

El Inventario de Cociente Emocional de Reuver Bar-On fue aplicado en los alumnos del Curso de Medicina de Animales de Compañía con el objetivo de detectar el nivel de desarrollo de sus habilidades cognitivas y emocionales, a fin de identificar e implementar las estrategias pedagógicas que podrían resultar más efectivas.

\section{MÉTOdos}

Los contenidos del curso están divididos en tres unidades didácticas: a) Medicina Interna I (6 semanas), que incluye medicina del oído y de los sistemas respiratorio y digestivo; b) Medicina Interna II (6 semanas), que incluye medicina de los sistemas reproductor, cardiovascular endocrino, urinario y dermatología; y c) Tópicos en Medicina (5 semanas), que incluye medicina del sistema nervioso, oncología, oftalmología y afecciones del sistema osteoarticular.

Las estrategias didácticas comprenden las siguientes metodologías: Clases teóricas de los temas consignados en el sílabo, con el fin de consolidar los conocimientos necesarios; Clases prácticas de procedimientos terapéuticos, diagnósticos clínicos rutinarios e imagenológicos con el fin de afianzar capacidades, habilidades y destrezas en la práctica clínica; Revisión de la información científica de tópicos asignados, a partir de la cual se elaboran informes que conforman las lecturas de temas para evaluaciones (pasos) cortas; y Trabajo de investigación clínica a partir de la casuística de la CVCH-FAVEZ, el cual es sustentado en el aula.

La evaluación comprendió las siguientes actividades:

-Exámenes Teóricos, que abarcan principalmente temas de desarrollo basados en casos clínicos y complementados con preguntas objetivas (alternativas múltiples, verdaderofalso, relacionar o elaboración de tablas) de acuerdo a las características del tema;

-Exámenes Prácticos, consistentes en la resolución de problemas o casos clínicos que permitan el reforzamiento en la realización de procedimientos terapéuticos, diagnósticos clínicos, imagenológicos o en la elaboración 
Cuadro 1. Áreas y descripciones de las habilidades cognitivas y emocionales evaluadas en el curso de Medicina de Animales de Compañía (Semestre 2012-II), Facultad de Medicina Veterinaria y Zootecnia, Universidad Peruana Cayetano Heredia, Lima

\begin{tabular}{ll}
\hline \multicolumn{1}{c}{ Habilidades Cognitivas } \\
\hline Área & Descripción \\
\hline Solución de problemas & $\begin{array}{l}\text { Habilidad para identificar y definir los problemas, como también } \\
\text { para generar e implementar soluciones efectivas. }\end{array}$ \\
Adaptabilidad & $\begin{array}{l}\text { Habilidad de la persona para establecer una relación de equilibrio } \\
\text { earente de conflictos con su ambiente social, físico y } \\
\text { emocional. }\end{array}$ \\
Flexibilidad & $\begin{array}{l}\text { Habilidad para realizar un ajuste adecuado de los pensamientos, } \\
\text { emociones y conductas a situaciones y condiciones cambiantes. }\end{array}$ \\
\hline Área & \multicolumn{1}{c}{ Habilidades Emocionales } \\
\hline Control de impulsos & $\begin{array}{l}\text { Habilidad para resistir o postergar un impulso o tentaciones para } \\
\text { actuar y controlar las emociones. }\end{array}$ \\
Manejo del estrés & $\begin{array}{l}\text { Habilidad para soportar eventos adversos, situaciones estresantes } \\
\text { y emociones fuertes sin derrotarse, enfrentando activa y } \\
\text { positivamente el estrés. } \\
\text { Habilidad para comprender, respetar y aceptarse a uno mismo, } \\
\text { tanto en los aspectos positivos como negativos, así como también } \\
\text { en las limitaciones y posibilidades. }\end{array}$ \\
\hline Autoconcepto & \multicolumn{1}{l}{} \\
\hline
\end{tabular}

de artículos de información científica realizados previamente en las clases prácticas, con el fin de afianzar y reforzar capacidades, destrezas y habilidades en la ejecución de los mismos;

-Revisión de Información Científica de Tópicos Asignados, que comprende la elaboración de un informe/resumen de hasta tres páginas sobre temas consignados en el sílabo, el cual es expuesto con ayuda audiovisual por grupos designados al azar;

-Pasos Cortos, realizados en los primeros 10 minutos de la clase práctica y comprenden las lecturas asignadas y la revisión de información científica de tópicos otorgados en las fechas consignadas en el sílabo;
- Caso Clínico, el cual es realizado a partir de la casuística de la $\mathrm{CVCH}$, donde los grupos preparan un caso realizando el seguimiento y las pruebas necesarias durante las 12 primeras semanas de clases;

-Informe del Caso Clínico, el cual es el resultado final de esta investigación clínica, entregada en versión electrónica en formato de artículo científico;

-Sustentación del Caso Clínico, entre las semanas 13 a 16 del semestre. Comprende la calidad expositiva, el conocimiento del tema y la capacidad de respuesta a las interrogantes. Además, los asistentes son evaluados mediante sus aportes, comentarios o preguntas adicionales que realizan durante la presentación del tema. 
Otras actividades formativas, no calificadas, consideran la participación en actividades de extensión social, donde se realiza apoyo de servicio veterinario en entidades nacionales o privadas, y la elaboración de videos de procedimientos clínicos publicados a través del Blog académico www.medanimcia.blogspot.com. Asimismo, la asistencia al servicio de atención clínica en $\mathrm{CVCH}$ FAVEZ, de forma voluntaria y rotativa, a fin de desarrollar conocimiento y experiencia a partir de casos de la práctica real e interactuar con médicos veterinarios de la práctica clínica y con los propietarios de las mascotas.

La evaluación de las habilidades cognitivas y emocionales de los alumnos del curso fue realizada por la Oficina de Tutoría Académica y Consejería Psicológica de la Dirección de la Carrera Profesional de Medicina Veterinaria y Zootecnia, adaptando la Encuesta del Inventario de Cociente Emocional de Reuver Bar-On (Ugarriza, 2001); y considerando los siguientes componentes de la Inteligencia Emocional: solución de problemas, adaptabilidad, flexibilidad, control de impulsos, manejo del estrés y autoconcepto (Cuadro 1).

La encuesta fue realizada en dos ocasiones: la primera en agosto de 2012 y la segunda en noviembre de 2012, como parte del Semestre 2012-II (agosto a diciembre). Los resultados de ambas encuestas se compararon para comprobar la efectividad de las estrategias pedagógicas empleadas.

Las habilidades cognitivas y emocionales se clasificaron como: Desarrolladas: personas con más probabilidades de sentirse satisfechas y eficaces en su vida, y con más capacidad para dominar los hábitos mentales que favorezcan su propia productividad, estabilidad y salud mental; Adecuadas: personas que demuestran ciertas cualidades requeridas para llevar a cabo determinadas misiones, teniendo éxitos y fracasos, de manera equilibrada; y Por mejorar: personas que no pueden poner cierto orden en su vida emo- cional, librando batallas que sabotean su capacidad de concentrarse en sus actividades y pensar con claridad (Dawda y Hart, 2000; Ugarriza, 2001; Bar-On et al., 2003).

\section{Resultados y Discusión}

El número de alumnos matriculados fue de 30 y el número de encuestados en ambas ocasiones fue de 24 . El resultado global de la primera encuesta muestra el 25,54 y $21 \%$ de los alumnos con habilidades cognitivas y emocionales clasificadas como desarrolladas, adecuadas y por mejorar, respectivamente; en tanto que en la segunda encuesta cambian los valores a 54, 33 y $13 \%$ para cada una de estas medidas. Se puede observar que el porcentaje de alumnos con habilidades desarrolladas se duplicó al final del curso, lo cual muestra un importante progreso en el desarrollo de estas habilidades (Fig. 1).

El Cuadro 2 muestra el resultado porcentual de los indicadores específicos de las habilidades cognitivas y emocionales. Estas habilidades son componentes factoriales de la inteligencia emocional que son evaluadas con el inventario; asimismo, estas habilidades y destrezas se desarrollan a través del tiempo, cambian a través de la vida y pueden mejorar con el entrenamiento (Ugarriza, 2001), como se puede apreciar en los resultados observados.

El número de alumnos con habilidades calificadas como desarrolladas se duplicó entre el inicio y fin del curso en las áreas de Solución de problemas, Control de impulsos y Autoconcepto, disminuyendo grandemente la cantidad de alumnos calificados 'por mejorar' en la segunda encuesta. En el área de Adaptabilidad, la mayoría mantuvo un nivel adecuado, en tanto que los alumnos que necesitaban mejorar disminuyeron marcadamente en la segunda encuesta. Por otro lado, el área de Flexibilidad se mantuvo relativamente estable. 


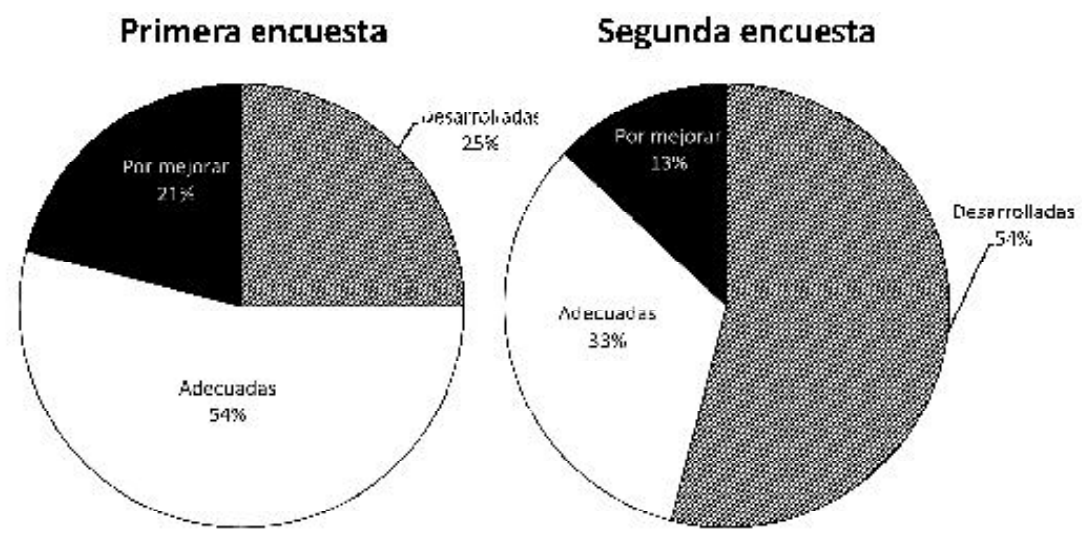

Figura 1. Clasificación del manejo de habilidades cognitivas y emocionales del resultado global de la evaluación de los alumnos del curso de Medicina de Animales de Compañía (Semestre 2012-II), Facultad de Medicina Veterinaria y Zootecnia, Universidad Peruana Cayetano Heredia, Lima, en base a dos encuestas (agosto y noviembre 2012)

Cuadro 2. Resultado porcentual de las clasificaciones de los indicadores específicos de las habilidades cognitivas y emocionales evaluadas en el curso de Medicina de Animales de Compañía (Semestre 2012-II), Facultad de Medicina Veterinaria y Zootecnia, Universidad Peruana Cayetano Heredia, Lima

\begin{tabular}{|c|c|c|c|c|c|c|}
\hline \multirow{2}{*}{ Habilidades } & \multicolumn{3}{|c|}{ Primera encuesta (\%) } & \multicolumn{3}{|c|}{ Segunda encuesta $(\%)$} \\
\hline & Desarrolladas & Adecuadas & Por mejorar & Desarrolladas & Adecuadas & Por mejorar \\
\hline $\begin{array}{l}\text { Solución de } \\
\text { problemas }\end{array}$ & 25 & 25 & 50 & 54 & 25 & 21 \\
\hline Adaptabilidad & 33 & 38 & 29 & 38 & 50 & 12 \\
\hline $\begin{array}{l}\text { Control de } \\
\text { impulsos }\end{array}$ & 17 & 29 & 54 & 42 & 33 & 25 \\
\hline Autoconcepto & 42 & 41 & 17 & 83 & 13 & 4 \\
\hline Flexibilidad & 21 & 37 & 42 & 17 & 50 & 33 \\
\hline $\begin{array}{l}\text { Manejo del } \\
\text { estrés }\end{array}$ & 29 & 29 & 42 & 12 & 21 & 67 \\
\hline
\end{tabular}

El área de Manejo de estrés mostró, evidentemente, un impacto negativo, pues el número de alumnos con habilidades desarrolladas o adecuadas disminuyó considerablemente entre la primera y segunda encuesta, lo cual puede deberse a que, si bien las nuevas estrategias pedagógicas fueron efectivas, estas demandaron mucho esfuerzo por parte de los alumnos. 
En cuanto a logros académicos, el 76.7\% (23/30) de alumnos aprobaron el curso con promedios de nota de 12.2 (rangos de 7.9 a 16.2), 11.3 (5.0 a 15.1$)$ y 11.5 (4.9 a 16.1$)$ para el primer, segundo y tercer capítulo, respectivamente. El promedio de nota final fue de 11.7 (rango de 6.0 a 15.8) sobre un máximo de 20, y que fueron promedios similares a este curso en años previos. Por otro lado, se observa un amplio rango de notas, donde el promedio quedó afectado por notas muy bajas y por la deserción de algunos alumnos.

Si bien las tres actividades no calificadas fueron demandantes de tiempo para el estudiante, y no conferían una posible mejora en los promedios académicos, permitieron, en cambio, el desarrollo de las habilidades cognitivas y emocionales. Extremera et al. (2007) han descrito que la mejora de estas habilidades permite que el estudiante presente menores niveles de agotamiento, mayor dedicación, menor percepción del estrés y absorción del desempeño, mejorando la calidad del aprendizaje e incrementando el bienestar psicológico del mismo.

Los estudios de Pérez y Castejón (2006) indican la independencia que existe entre la inteligencia emocional y el cociente intelectual; no obstante, indican asimismo que existen correlaciones entre la inteligencia emocional y algunos indicadores de rendimiento académico. Por tanto, valores como el espíritu humanista, el altruismo, la responsabilidad social y el interés en el voluntariado en esta área de la carrera permiten el desarrollo de las capacidades cognitivas y emocionales. Pendersen (2013) resalta, por su parte, la importancia del análisis de las emociones, la educación, y las relaciones humano-animales en la formación del Médico Veterinario y la exploración del afecto y la emoción como un modo didáctico particular.

El deseo de compartir conocimientos con personas que lo necesiten, el cuidado y amor desinteresado hacia los animales y la adquisición de experiencias socio-emociona- les son las fuentes para el desarrollo de estas capacidades y herramientas que serán útiles para el futuro profesional; siendo más satisfactorio el enriquecimiento del espíritu y la vida interior del estudiante que la misma calificación, lo cual permite la formación integral del ser humano en un contexto social real (Folgueiras y Martínez, 2009).

Extremera y Fernández-Berrocal (2003) mencionan la necesidad de desarrollar programas de aprendizaje socio-emocional basados en la capacidad de percibir, comprender y regular emociones, mientras que Shaw et al. (2010) describen el desarrollo de las habilidades de comunicación en veterinarios de práctica privada para mejorar el desempeño de la comunicación con sus clientes. Este mismo efecto se buscó al fomentar la asistencia al servicio de atención $\mathrm{CVCH}$ de los estudiantes, permitiendo la interacción con profesionales de su área, propietarios y pacientes.

Se dispone de escasa literatura sobre el diseño, aplicación y evaluación de programas de intervención centrados en la adquisición de habilidades y competencias de la inteligencia emocional a nivel de Latinoamérica (Pena y Repetto, 2008) y en el país (Ugarriza, 2001; Ugarriza y Pajares, 2008), por lo que este estudio pretende dar una primera visión e iniciar programas de intervención con estrategias pedagógicas que fomentan el desarrollo personal y que faciliten el acceso al mundo laboral del egresado de la carrera profesional de Medicina Veterinaria y Zootecnia.

\section{Literatura Citada}

\section{Andreu MA, Gonzales JA, Labrador MJ, Quintanilla I, Ruiz T. 2004. Mé- todo del caso, ficha descriptiva y nece- sidades. España: Universidad Politécnica de Valencia - Grupo de Metodologías Activas (GIMA-UPV). 22 p.}


2. Baéz EN, Mendoza D, Ramírez HC. 2006. Desarrollo de competencias TIC. México: Universidad Tecnológica de Nezahualcóyotl. 16 p.

3. Bar-On R. 1997. The emotional quotient inventory (EQ-i). A test of emotional intelligence. Toronto, Canada: MultiHealth Systems.

4. Bar-On R, Tranel D, Denburg NL, Bechara A. 2003. Exploring the neurological substrate of emotional and social intelligence. Brain 126: 1790-1800.

5. Cortés JF, Barragán C, Vásquez ML. 2002. Perfil de inteligencia emocional: construcción, validez y confiabilidad. Salud Mental 25(5): 50-60.

6. Dawda D, Hart SD. 2000. Assessing emotional intelligence: reliability and validity of the Bar-On Emotional Quotient Inventory (EQ-i) in university students. Pers Indiv Differ 28: 797-812.

7. Dodd L. 2007. The impact of problembased learning on the information behavior and literacy of veterinary medicine students at University College Dublin. J Acad Libr 33: 206-216.

8. Extremera N, Durán A, Rey L. 2007. Inteligencia emocional y su relación con los niveles de burnout, engagement y estrés en estudiantes universitarios. Rev Educ 342: 239-256.

9. Extremera N, Fernández-Berrocal P. 2003. La inteligencia emocional en el contexto educativo: hallazgos científicos de sus efectos en el aula. Rev Educ 332: 97-116.

10. Extremera N, Fernández-Berrocal P, Mestre JM, Guil R. 2004. Medidas de evaluación de inteligencia emocional. Rev Latinoam Psicol 36: 209-228.

11. Folgueiras P, Martínez M. 2009. El desarrollo de las competencias en la universidad a través del aprendizaje y servicio solidario. RIED 2(1): 56-76.

12. Kennedy D. 2007. Redactar y utilizar resultados en el aprendizaje. Irlanda:
University College Cork, Quality Promotion Unit. 103 p.

13. Larraín AM, González LE. (s.f.). Formación universitaria por competencias. [Internet], [20 diciembre 2012]. Disponible en: http://sicevaes.csuca.org/ attachments/134_Formacion\%20 Universitaria\%20por\%-20competencias.PDF

14. Pena M, Repetto E. 2008. Estado de la investigación en España sobre inteligencia emocional en el ámbito educativo. Electron J Res Educ Psych 6: 401420.

15. Pendersen H. 2013. Parasitic pedagogies and materialities of affect in veterinary education. Emotion Space Society. [Internet]. Disponible en: http:// dx.doi.org/10.1016/j.emospa.2013.10.002

16. Pérez N, Castejón JL. 2006. Relación entre la inteligencia emocional y el cociente intelectual con el rendimiento académico en estudiantes universitarios. REME 22. [Internet]. Disponible en: http://reme.uji.es/articulos/numero22/ article $6 /$ numero $\% 2022 \% 20$ article\%206\%20RELACIONS.pdf

17. Shaw JR, Barley GE, Hill, AE, Larson $S$, Roter DL. 2010. Communication skill education onsite in a veterinary practice. Patient Educ Couns 80: 337-344.

18. Ugarriza N. 2001. La evaluación de la inteligencia emocional a través del Inventario de Cociente Emocional BarOn (I-CE) en una muestra de Lima Metropolitana. Persona 4: 129-160.

19. Ugarriza N, Pajares L. 2008. La evaluación de la inteligencia emocional a través del inventario de BarOn ICE: NA, en una muestra de niños y adolecentes. Persona 8: 11-58

20. Yoo M-S, Park J-H. 2014. Effect of case-based learning on the development of graduate nurses' problem-solving ability. Nurse Educ Today 34: 47-51. 